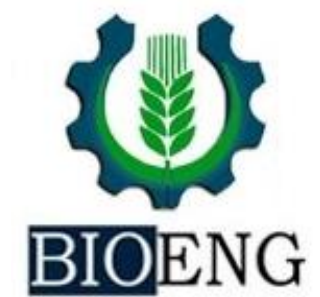

\section{UMA DÉCADA DA ENGENHARIA DE BIOSSISTEMAS: DESCRIÇÃO INSTITUCIONAL E NÃO INSTITUCIONAL DO CURSO}

\author{
L. P. Magalhães $1 *$
}

${ }^{1}$ Escola Superior de Agricultura Luiz de Queiróz, Departamento de Engenharia de Biossistemas, USP, Piracicaba, São Paulo, Brasil.

Article history: Received 31 August 2020; Received in revised form 25 September; Accepted 30 September; Available online 30 December 2020.

\title{
RESUMO
}

Após mais de 10 anos da criação do primeiro curso de Engenharia de Biossistemas no Brasil, será que já é possível obter uma definição (ou descrição) única da Engenharia de Biossistemas? As descrições contidas nos sites institucionais das quatro Universidades oferecem uma descrição similar sobre o curso? Quais palavras-chave esses sites apresentam na descrição da Engenharia de Biossistemas? E os sites não-institucionais que descrevem a profissão, se utilizam de quais palavras com mais frequência? Para responder estas perguntas foi feita uma mineração nos textos utilizados pelas quatro Universidades na descrição do curso, para assim obter as palavras mais utilizadas na caracterização do curso, seu grafo de relação e a similaridade entre as descrições. Os resultados mostraram baixa similaridade entre os textos, o enfoque na palavra 'produção' como a mais citada e que os textos nãoinstitucionais apresentam mais o termo 'agricultura' como central do que aqueles institucionais.

Palavras-chave: Mineração de Textos. Processamento de Linguagem Natural. Grafos. Python.

\section{A DECADE OF BIOSYSTEM ENGINEERING: INSTITUTIONAL AND NON INSTITUTIONAL DESCRIPTION OF THE COURSE.}

\begin{abstract}
After more than 10 years of the creation of the first Biosystems Engineering course in Brazil, is it possible to obtain a unique definition (or description) of Biosystems Engineering? Do the descriptions on the institutional websites of the four Universities offer a similar description about the course? What keywords do these sites present in the description of Biosystems Engineering? And which non-institutional websites that describe the profession, use which words most often? To answer these questions, the texts used by the four Universities in the description of the course were mined to obtain the most used words in the characterization of the course, its relation graph and the similarity between the descriptions. The results showed
\end{abstract}

\footnotetext{
*1eonardo.magalhaes@usp.br
} 
low similarity between the texts, the focus on the word 'production' as the most cited and that non-institutional texts have the term 'agriculture' more central than institutional ones.

Keywords: Text Mining. Natural Language Processing. Graphs. Python.

\section{INTRODUÇÃO}

A Engenharia de Biossistemas se inicia no Brasil, enquanto curso de graduação, no ano de 2009 na Universidade de São Paulo em Pirassununga. Apesar de em nosso País este curso ter sido criado apenas na última década, nos EUA o movimento por uma Engenharia que buscasse compreender as relações matemáticas dos processos biológicos com os físicos se inicia em 1960. Segundo TAO et al. (2006), em reunião da então ASAE (American Society of Agricultural Engineering), George Washington Giles propõe que a Engenharia Agrícola deve se atentar aos processos biológicos envolvidos na produção e não apenas às práticas de engenharia. Nesta fala ele aponta que: "O núcleo de nossa profissão deve ser construído sobre as leis de engenharia que governam os processos complexos e intrincados de plantas e animais. Isso é o que distingue a Engenharia Agrícola de outras profissões da engenharia". LOEWER (1997) aponta que a proposição de Giles foi aceita pela ASAE, mas rejeitada pelo público principal da convenção (essencialmente composto pelos representantes de indústrias do setor). Ainda segundo TAO et al. (2006), em 1987 departamentos de Engenharia Agrícola começam a estabelecer mudanças nos currículos de graduação tendo como núcleo dois pontos: os currículos deveriam ser baseados nas ciências biológicas para que os engenheiros obtivessem conhecimento da engenharia em associação com a biologia; e o currículo base deveria dar a capacidade de que os graduandos pudessem atender às necessidades de mudança da sociedade para a engenharia relacionada à sistemas biológicos. Em 2005 a ASAE então modifica seu nome para ASABE
(American Society of Agricultural and Biological Engineerings) por entendimento de seus membros de que o novo nome representaria melhor as designações da profissão. Entre os diferentes nomes que englobam a Engenharia Biológica, como a Bioengenharia ou Engenharia Bioquímica, se encontra a Engenharia de Biossistemas, mudando o nome de acordo com o enfoque a que se necessita dar ao curso. Em 1995, 49\% dos Departamentos de Engenharia Agrícola dos Estados Unidos da América (EUA) tinham modificado seus nomes para algum que contivesse biológicos/biossistemas ou biorecursos (CUELLO, 1995). Em 2005 esse número já era de $85 \%$ (YOUNG, 2006). A China, devido às mudanças socioeconômicas a que o País passou e em especial a maior abertura econômica dos últimos 20 anos, iniciou nos anos 2000 a mudança dos currículos nos cursos de Engenharia Agrícola. Neste ano, na Universidade de Zhejiang, foi criado o primeiro curso de Engenharia de Biossistemas, unindo engenharia e biologia (HE et al., 2007). Este curso criado possui forte base em Engenharia Aplicada (correspondendo a $24,3 \%$ do curso), educação geral $(22,15 \%)$ e matemática $(10,5 \%)$. Apesar dos autores citarem a aliança entre engenharia $e$ biologia, as disciplinas de biologia correspondem a $8 \%$ dos créditos.

De acordo com definição da ASABE, então a Engenharia Agrícola e Biológica é a, em tradução livre: "disciplina da engenharia que aplica os princípios da engenharia e os conceitos fundamentais da biologia aos sistemas e ferramentas agrícolas e biológicos, variando em escala do nível molecular ao ecossistema, para a produção, processamento e gestão segura, 
eficiente e ambientalmente sensível dos sistemas agrícolas, biológicos, alimentares e de recursos naturais" (ASAE, 2020). Sendo assim, em mais de dez anos de sua criação no Brasil, como se estabelece a definição do curso nas instituições que o oferecem? Já se consegue estabelecer uma definição única do curso? E nos meios não institucionais de divulgação, como é delineado o campo de atuação da Engenharia de Biossistemas?

Nesse período, desde o início do curso no País, se estabeleceram descrições acerca do curso nos endereços eletrônicos oficiais (institucionais) das Universidades e Faculdades onde é ministrado o curso. Nestes textos estão contidas informações sobre como cada instituição visualiza, ou compreende, a organização do curso e suas áreas de atuação. Estas informações, portanto, podem ser mineradas para análise mais aprofundada desta imagem construída. A mineração de textos é a recuperação de informações, extração de informações textuais, usando técnicas para posterior análise através do processamento de linguagem natural, mineração de dados, aprendizagem de máquina $\mathrm{e}$ desenvolvimento de algoritmos (HOTHO et al., 2005 apud DUVAL \& SILVA, 2019).

\section{MATERIAIS E MÉTODOS}

Inicialmente se procedeu a coleta dos textos para análise. Foram escolhidas as descrições sobre a Engenharia de Biossistemas contidas nos endereços eletrônicos oficiais da Faculdade de Zootecnia e Engenharia de Alimentos (FZEA) da USP, do Centro de Desenvolvimento Sustentável do Semiárido (CDSA) da UFCG, da Faculdade de Ciências e Engenharia da UNESP em Tupã e do Instituto Federal de São Paulo em Avaré. A Universidade de São Paulo (USP) também possui uma descrição acerca do curso em um site chamado "USP e as Profissões", sendo
Machado-da-Silva \& Nogueira (2001) estabelecem que na construção social da realidade os entendimentos acerca de uma organização podem ser, ou não, convergentes. Eles dizem também que "grupos coesos crescem em torno de entendimentos comuns; grupos fragmentados esposam visões de realidade múltiplas". Assim, a descrição realizada acerca da Engenharia de Biossistemas pelas diferentes instituições é, ou não, convergente? E os meios nãoinstitucionais, que descrevem a Engenharia de Biossistemas, possuem visão convergente, ou ao menos uma descrição semelhante sobre o curso?

Tendo esses fatos em vista, o objetivo deste trabalho é avaliar como se dá a descrição da Engenharia de Biossistemas nos meios institucionais e nãoinstitucionais, qual imagem passam ao leitor ao tentar entender o que é, qual campo de atuação e a estrutura da Engenharia de Biossistemas no Brasil. E ainda comparar se as palavras mais usadas coincidem com as dissertações de Mestrado que estes profissionais já publicaram.

então essa descrição também escolhida. $\mathrm{O}$ mesmo ocorre com a Universidade Federal de Campina Grande (UFCG) que além da descrição do CDSA tem outra em seu site geral. Quanto às descrições não institucionais, foram selecionadas aquelas presentes nos seguintes sites: Wikipedia, Guia do Estudante, Guia da Carreira, Engenharia 360, Engenharia é, Vai de Bolsa e Globo Rural. Estes sites foram escolhidos por terem grande fluxo de acesso e serem os que aparecem nas primeiras posições de pesquisas realizadas em sites de busca. Assim, totalizam-se seis descrições institucionais e sete não- 
institucionais para coleta dos textos e comparação. Para avaliar a descrição da Engenharia de Biossistemas fora do Brasil foram selecionadas a descrição do site oficial da ASABE e da Wikipedia em inglês.

Em seguida, para cada texto, em um algoritmo desenvolvido em Python foram calculadas as palavras mais frequentes e em cada um é construída a nuvem de palavras dos textos. Este algoritmo utiliza a biblioteca ntlk (de processamento natural de linguagem) para separar o texto em sentenças e palavras. A partir disto, é verificado a cada sentença quais palavras são mais repetidas ao longo do texto de entrada. Como as descrições institucionais do curso não são grandes (entre dois e três parágrafos) se estabeleceu que seriam selecionadas as cinco palavras mais frequentes para representarem como palavras-chave cada descrição. Para evitar que palavras como conjunções e conectivos aparecessem como palavraschave, foi feita uma limpeza em cada texto das chamadas stopwords (como exemplo, foram retiradas as palavras para, e, ou, também as pontuações entre outras).

Para uma segunda análise, utilizando o algoritmo, novamente em linguagem Python foi feita uma comparação de similaridade entre os diferentes textos.

\section{RESULTADOS E DISCUSSÃO}

Após a primeira análise, se pôde verificar as palavras mais frequentes em cada texto. $\mathrm{Na}$ descrição presente no site da FZEA se verifica que as cinco mais repetidas foram: 'produção', 'biossistemas', 'profissional', 'engenharia' e 'agrícola'. Na Figura 1 se observa a nuvem destas palavras mais citadas. Palavras semelhantes às mais
Nesta análise é calculado um vetor com as palavras-chave de cada texto, sendo a similaridade o valor do cosseno entre os vetores de cada texto.

Uma terceira análise, agora utilizando as bibliotecas nltk e networkx do Python, procurou-se estabelecer os grafos de conexão entre as principais palavras. Por se tratarem de textos curtos, nesta fase da análise, os textos foram unidos em apenas um. Assim, as descrições institucionais do curso formaram o texto número um e as não-institucionais o texto dois. Para tal, foram selecionadas as 100 palavras mais frequentes e avaliado o quanto cada uma aparece relacionada às demais. Por exemplo, a palavra 'engenharia' em cada sentença se liga com quais outras palavras dentre as mais frequentes. Com isso, se espera estabelecer uma espécie de resumo da descrição institucional do curso, estabelecendo quais palavras são centrais desse discurso e como se conectam e então comparar com a descrição feita por outros meios que não sejam oficiais das Universidades. Através da repetição de palavras e da relação das palavras com aquelas consideradas chave no texto é possível estabelecer um grafo com a rede de palavras (FERGUSON \& LOVELL, 2014). Todos os algoritmos criados podem ser acessados por meio do link*.

frequentes no site "USP e as Profissões" que foram: 'produção', 'formação', 'engenheiro', 'biossistemas', 'profissional'. Sendo que, neste caso a descrição feita pela USP destacou a palavra formação e ao invés de engenharia aparece a palavra engenheiro. 
Figura 1. Nuvem de palavras da descrição institucional da FZEA.

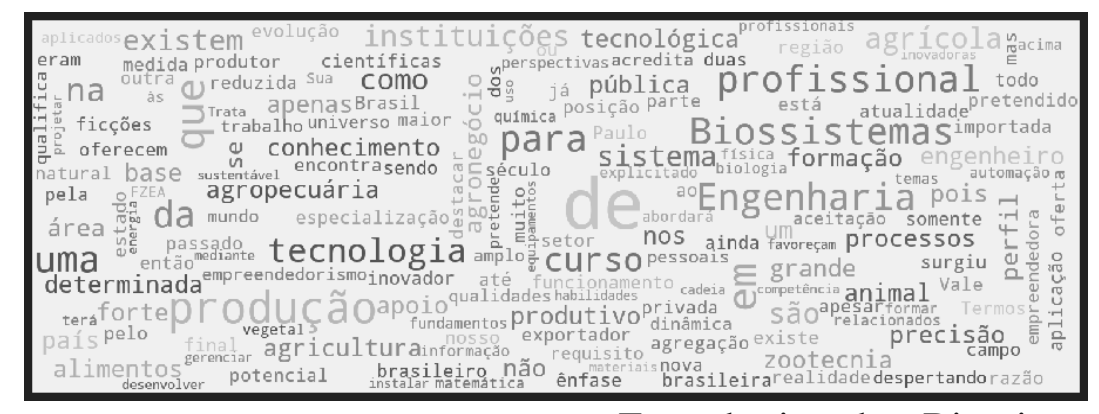

Em relação à UFCG, a descrição no site do CDSA, as palavras mais frequentes foram: 'engenharia', 'curso', 'biossistemas', 'produção' e 'fibras'. Da mesma Universidade, mas agora com texto disponível no Portal da Universidade, as palavras mais citadas são: 'biossistemas', 'energia', 'produtos', 'produção' e 'engenharia'. Em relação às descrições feitas pela USP, se nota nos textos da UFCG que a palavra produção é repetida novamente entre as mais frequentes. Tal fato se repete na descrição feita pela UNESP ('produção', 'biossistemas', 'automação', 'demanda' e 'animal') e pelo IFSP ('produção', 'engenharia', 'biossistemas', 'agropecuária', 'fibras').

No texto da FZEA a palavra produção aparece sete vezes, no "USP e as profissões" se repete seis vezes, duas vezes no texto da UFCG/CDSA, quatro vezes no portal da UFCG, cinco vezes na UNESP e três na descrição do IFSP.

No dicionário se encontra a definição de produção como: "primeiro estágio de uma série de processos econômicos que levam bens e serviços às pessoas" ou "resultado de algum processo humano" (in: DICIO, 2020). Pelas definições, e repetição da palavra nas seis descrições, se nota que a Engenharia de Biossistemas tem como um de seus objetivos levar bens e serviços à sociedade (pessoas). Mas, que tipo de bens e serviços? Entre as diferentes definições, de cada Universidade, são percebidas palavras como: agrícola, fibras, energia, animal e agropecuária. Assim, se depreende, que nas definições, a
Engenharia de Biossistemas foca em produzir bens dentro da área agropecuária, reforçado pelas palavras anteriormente citadas.

Porém, o enfoque dentre esses bens se modifica de acordo com cada Universidade e a realidade em que cada uma se insere. Por exemplo, na UFCG a produção de fibras e energia recebe destaque, nas descrições do CDSA e do site geral da Universidade, respectivamente. Apesar, de se situar em uma Faculdade que possui foco na produção animal, este termo não aparece entre os mais citados na descrição da FZEA, o que se diferencia da UNESP que a cita mais vezes. Apesar de que nesta última a palavra se repita três vezes, enquanto na descrição feita pela FZEA aparece duas vezes.

O enfoque na palavra produção também é visto nas descrições não institucionais do curso. Ela aparece entre as cinco mais citadas nos sites: Guia do Estudante, Engenharia 360, Guia da Carreira, Engenharia é, bem como na Wikipédia em português. No site Vai de Bolsa ela não aparece entre as cinco, mas quando se amplia para as dez palavras mais citadas, ela está entre estas. Apenas na descrição feita pelo Globo Rural esta palavra não é está entre as mais citadas.

Nas descrições não-institucionais também se começa a destacar, ou delimitar mais, o campo de atuação desta profissão. A palavra agropecuária, que aparece entre as mais citadas apenas na descrição do IFSP entre as institucionais, se encontra entre as 
cinco mais repetidas pelos sites Guia do Estudante e Engenharia 360. Por serem textos maiores, contidos nestes sites, se ampliamos para as dez palavras mais citadas (e que aparecem mais de uma vez) esta palavra também é bastante citada nos sites do Globo Rural e Guia da Carreira. Nas descrições institucionais em que agropecuária aparece mais de uma vez são: FZEA (2) e USP e as profissões (3, mas uma vez como a similar agropecuários). Nas demais aparece uma vez (CDSA) ou nenhuma (UNESP e UFCG).

Dentre as palavras mais citadas, tanto nas descrições, como nas institucionais, não aparece a palavra sustentabilidade (nem palavras correlatas). No site da FZEA 'sustentável' é citada apenas uma vez na descrição do curso, igualmente no site "USP e as profissões", uma vez (como sustentada) na descrição feita pelo CDSA, duas vezes (sustentável e sustentada) no portal da UFCG, nenhuma vez na descrição feita pela UNESP e uma vez pelo IFSP. Apesar de aparecer poucas vezes, e em menor quantidade do que a palavra produção, dentro dos objetivos da Engenharia de Biossistemas, a sustentabilidade têm importância na sua atuação. Fato este evidenciado na descrição do escopo de trabalhos do "Journal of Biosystems Engineering", principal publicação da área e uma das principais revistas de pesquisa agrícola. A definição do escopo da revista destaca que ela publica "pesquisas em engenharia e ciências físicas que representam avanços na compreensão ou modelagem do desempenho de sistemas biológicos para desenvolvimento sustentável no uso da terra e no meio ambiente, agricultura e amenidades, processos de bioprodução e cadeia alimentar". Esta visão, diferente da apresentada nacionalmente, também é notada nas definições da Engenharia de Biossistemas encontradas na Wikipédia em inglês e naquela definida pela ASABE. Nelas, entre as cinco mais citadas, aparecem palavras relacionadas ao meio- ambiente e sustentabilidade como diretamente 'meio-ambiente' na Wikipédia (três vezes) e 'seguro' (três vezes) na ASABE. Sendo que, 'seguro' na definição da ASABE se relaciona com alimentos, produção e ambiente. Para comparação, na Wikipédia em português, meio-ambiente não é citado em nenhuma vez.

Apesar de certo preconceito com as informações contidas em páginas da Wikipédia, na busca por informações acerca da Engenharia de Biossistemas, é a página que aparece no topo das buscas. A página da Engenharia de Biossistemas na Wikipédia (em estatísticas obtidas no site), entre Julho de 2015 (data disponível para início da pesquisa de visitas) e Julho de 2020 recebeu 6285 visitas, uma média mensal de 103 visitantes (VISUALIZAÇÕES, 2020). Estes números demonstram a importância desta página para divulgação do curso e criação de uma descrição única para a profissão. Possui também a vantagem de ser uma ferramenta aberta, o que possibilita que funcionários e professores das Universidades onde a Engenharia de Biossistemas é ofertada contribuam com referências confiáveis para definição do curso.

Em relação à esta definição do que se trata o curso, na avaliação da similaridade entre os textos disponíveis, o maior valor foi entre a descrição da FZEA e da "USP e as profissões" $(0,525)$. Em seguida, a maior similaridade foi entre o texto do CDSA e da UFCG (0,315). Tanto no caso da FZEA e USP, quanto do CDSA e UFCG, por se tratarem de descrições feitas por instâncias da mesma instituição, se explica a maior similaridade nos dois casos. Entre diferentes instituições a maior similaridade calculada foi entre a FZEA e CDSA, contemporâneos no ano de criação do curso. Entre os textos institucionais e os de outros sites, a maior similaridade foi entre o descrito pelo Guia do Estudante e a FZEA, com uma similaridade de 0,314. Fato este que ocorre pelo texto do Guia ter 
sido escrito com consulta ao coordenador de curso da FZEA na época de publicação do mesmo. Em relação à Wikipedia, o texto de descrição da Engenharia de Biossistemas tem mais similaridade com o produzido pelo IFSP $(0,230)$ e em seguida pelo da FZEA $(0,218)$.

A baixa similaridade entre os textos avaliados (cujo valor máximo seria igual a um) é coerente com o encontrado por Silva \& Nunes (2016). Estes autores avaliaram as redes de colaboração entre pesquisadores da Engenharia de Biossistemas e encontraram uma densidade de grafo de 0,025 (cujo valor ia até um). Isto indica baixa colaboração entre os pesquisadores avaliados, e pode indicar baixa colaboração em outras áreas como em elaborar uma descrição única do significado da Engenharia de Biossistemas, tendo em vista a baixa similaridade entre os textos dos sites oficiais.

Para tentar estabelecer a definição da Engenharia de Biossistemas, ou ao menos um indicativo dos campos de atuação destes engenheiros, e se as descrições institucionais refletem na atuação profissional deles, foram avaliadas as dissertações já publicadas por engenheiros de biossistemas. Dentro deste escopo, foram encontrados 33 formandos da FZEA/USP que cumpriam esses requisitos. Os Programas de Pós-Graduação em que os mesmos se inserem vão desde Engenharia Agrícola até Zootecnia, passando por Engenharia Mecânica, de Alimentos e também de Materiais. Destaca-se que, apesar de não constar como palavra-chave em nenhuma descrição institucional do curso, oito formandos foram para pós-graduação na área de materiais. Esse fato pode ser explicado devido ao fato deste Programa em Engenharia de Materiais ser oferecido na mesma Faculdade em que esses formandos se titularam em Engenharia de Biossistemas. Porém, indica também que é uma área em destaque nas pesquisas da Engenharia de Biossistemas e deveria ser mais citada na descrição do curso.

Entre os 33, 14 já tinham publicado a dissertação de Mestrado, sendo selecionadas as que foram publicadas em Português, ou que tinham ao menos um resumo nesta língua, para que a tradução não afetasse o sentido das palavras selecionadas. Os resumos das dissertações foram copiados e incluídos em um texto único para obtenção das palavras mais citadas. Sendo selecionadas as 20 mais frequentes, que foram: 'produção', 'dados', 'efluente', 'resultados', 'térmico', 'minitomateiro', 'valores', 'água', 'estudo', 'diferentes', 'forma', 'solo', 'irrigação', 'sistema', 'três', 'área', 'produtividade', 'apresentaram', 'trabalho' e 'sendo'.

Novamente a palavra 'produção' se destaca entre as mais citadas, indicando que o enfoque nesse quesito se repete na pesquisa pós-formatura destes profissionais. Mas, outras palavras que não tiveram destaque nas descrições institucionais do curso, agora ganham destaque como: irrigação, solo, térmico, dados e produtividade. O termo dados possui relevância recente em pesquisas e aplicações tecnológicas, o avanço na capacidade computacional permitiu a análise de grande volume de dados (big data). Segundo HAN et al. (2012), o termo big data não se refere apenas à coleta e avaliação de dados, bem como a tomada de decisões em seguida. Na agricultura, onde são produzidos grandes volumes de dados e se necessita cada vez mais de tomada de decisão em função destes dados, o termo deve ganhar ainda mais destaque na Engenharia de Biossistemas. 
Figura 2. Grafo de relação entre as cem palavras mais citadas nas descrições institucionais

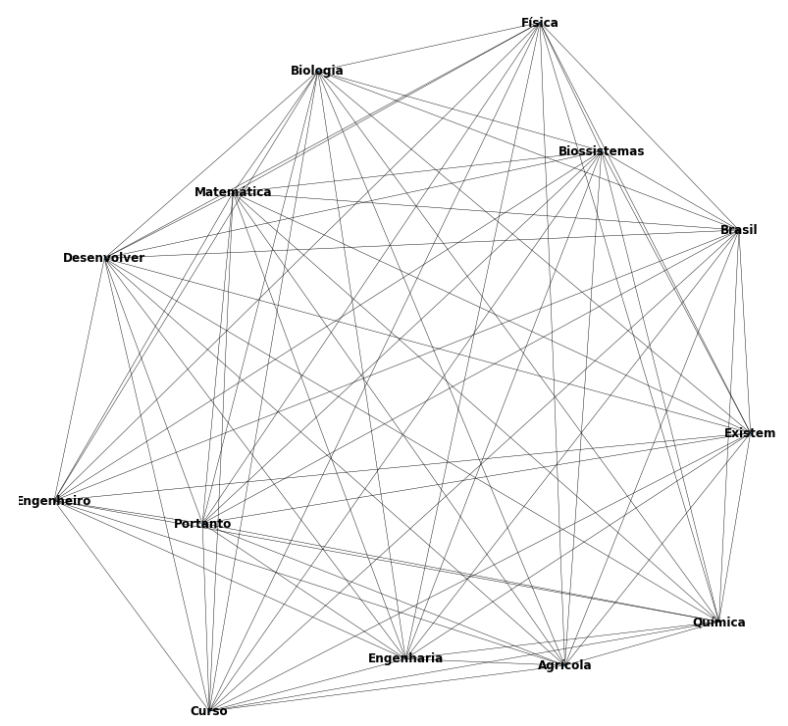

A palavra 'efluente' também aparece aqui, mesmo não sendo citada em nenhuma das descrições avaliadas sobre o curso. Três das dissertações avaliadas tinham este tópico como tema. Já em relação a 'minitomateiro', que aparece em destaque em duas dissertações das 14 avaliadas, por ser um tema muito específico não necessariamente reflete um campo de atuação, ou tema de interesse geral de pesquisa, mas se insere na temática de pesquisa na área vegetal, esta sim bastante citada nas descrições do curso.

Ao analisar como os termos chave se relacionam em cada tipo de descrição do curso, foram produzidos os grafos com as 100 palavras que mais aparecem na descrição institucional do curso, bem como naquelas produzidas por sites e blogs não institucionais.

Figura 3. Grafo de relação entre as cem palavras mais citadas nas descrições não institucionais

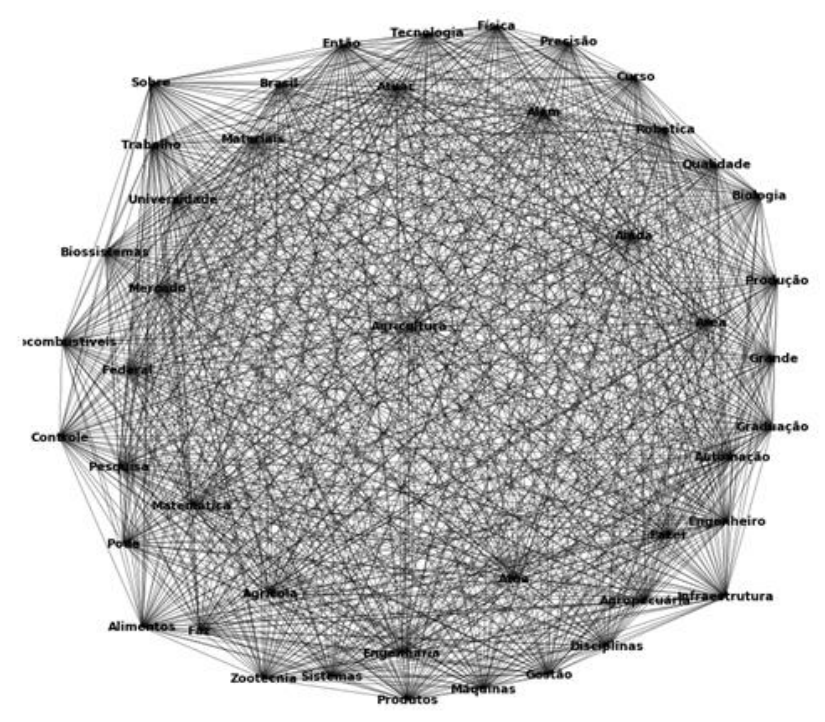


No grafo aparecem só as palavras, entre as 100 mais, que também têm forte relação entre si. A Figura 2 mostra o grafo das palavras presentes em todos os textos institucionais. Se pode notar que não há uma palavra central no grafo, todas as palavras se relacionam entre si e aparecem entre outras : matemática, física, química e biologia. Estas palavras se relacionam mais com a formação básica do curso, com os anos iniciais do mesmo, assim se pode inferir que as descrições realizadas pelas instituições de ensino focam em descrever mais os aspectos gerais do curso, ou refletir uma descrição muito focada na formação formal do curso e não na delimitação profissional do mesmo. Alguns destes textos institucionais foram produzidos no início da implantação do curso no País, quando ainda não haviam formados e não se produzia conteúdo acerca da Engenharia de Biossistemas. Como exemplo, apenas na descrição feita pela UNESP a palavra 'automação' aparece entre as mais citadas, refletindo uma visão mais recente do curso (pós 2014) que já tinha mais clara a atuação tecnológica destes profissionais.

Já pela Figura 3 se nota que os textos não institucionais do curso são maiores, com maior citação de palavras, o que se reflete em um grafo maior e mais interrelacionado. Outro ponto, a palavra 'agricultura' se apresenta no centro do grafo, o que representa que esta tem centralidade nestes textos. Essas descrições não institucionais já partem mais da ideia de que a atuação na agricultura é o objetivo central da Engenharia de Biossistemas. Ainda se percebe, que mesmo de maneira menos central nestes textos, as palavras 'tecnologia', 'zootecnia', 'precisão' e outras que não têm destaque nos textos institucionais aparecem neste grafo. Isso pode refletir tanto uma visão mais atualizada sobre o curso, com estes textos sendo produzidos quando o curso já estava mais consolidado em mais de uma Universidade, quanto uma visão menos restrita ao aspecto de formação (currículo) do curso e mais na atuação profissional.

Nunes \& Silva (2014) afirmam que há ainda ambiguidade na definição do que seria a Engenharia de Biossistemas. Para estes autores ela seria, para algumas pessoas, um sinônimo de Engenharia Biológica, mas sendo focada em seres vivos não-humanos. Mas, alguns temas como automação agrícola, irrigação e agricultura de precisão entrariam no escopo desta Engenharia, apesar de nestes campos ocorrer um sombreamento com outras engenharias, em especial a Agrícola e não receberem destaque nas definições da Engenharia de Biossistemas. Tal fato se justifica pela origem da Engenharia de Biossistemas, que derivou de Departamentos de Engenharia Agrícola, e nas palavras utilizadas para descrever o novo curso isto se reforça no aparecimento dos termos 'agrícola' e outras palavras (como 'vegetal','fibras' etc.) que se relacionam com a Engenharia Agrícola.

Apesar da forte presença de disciplinas como Eletrônica, Automação, Programação e outras, estes termos não se destacam nas descrições institucionais do curso, visto que não aparecem em geral nas mais citadas. $O$ que reforça a constatação de Nunes \& Silva (2014) destes termos ficarem mais à sombra.

Estes autores ainda citam um novo marco que poderia ajudar na definição desta nova área, a mudança do nome do Journal of Agricultural Engineering Research em 2001 para Journal of Biosystems Engineering. No primeiro editorial, após esta mudança de nomenclatura, a revista cita que a mudança se deu em função das mudanças da agricultura nos últimos 50 anos. A substituição da agricultura como principal produto da economia (nos EUA), e consequente industrialização da mesma, as preocupações com o aquecimento 
global e degradação de ecossistemas, a mudança nas formas de produção entre outros aspectos aumentaram o escopo da revista para além da agricultura. Porém, quando se olha para as palavras-chave nas descrições do curso aqui no Brasil (tanto institucionais quanto não) palavras referentes às mudanças climáticas não aparecem, e acerca da industrialização da agricultura aparecem de maneira indireta (através da palavra produção) e não do surgimento de novas tecnologias (como sensoriamento remoto, automação, visão computacional etc.).

A falta de uma definição mais clara acerca do curso, bem como da baixa divulgação, pode refletir no interesse acerca do curso
(Tabela 1). Avaliando os dados sobre os últimos cinco vestibulares deste curso, apenas para USP e UNESP, mostram que nas vagas oferecidas via FUVEST e VUNESP, respectivamente, têm em média uma relação candidato/vaga de 4,04 e 2,6, respectivamente. E que estes valores variam, ano a ano, a depender da relação do vestibular anterior. Ou seja, quando a relação aumenta, no ano seguinte cai a procura pelo curso, isto ocorrendo em especial no Vestibular da USP. Tal fato, pode indicar que a procura pelo curso se dá em função da dificuldade de entrada (menor relação candidato/vaga) do que em função de conhecimento sobre o curso.

Tabela 1. Relação Candidato/Vaga para USP e UNESP

\begin{tabular}{lccccc}
\hline 2016 & 2017 & 2018 & 2019 & 2020 \\
\hline USP & 7,80 & 2,59 & 5,04 & 2,83 & 1,97 \\
\hline UNESP & 2,8 & 2.5 & 2,8 & 3,00 & 1,90 \\
\hline
\end{tabular}

Dados obtidos dos sites oficiais de cada vestibular.

\section{CONCLUSÕES}

Pelos resultados se nota que ainda não há formação de uma definição única da Engenharia de Biossistemas, fato observado pela baixa similaridade entre textos institucionais descritivos do curso. As palavras de enfoque em cada descrição também se diferenciam. Em dissertações publicadas por Engenheiros de Biossistemas, aparecem palavras que não possuem destaque nas descrições institucionais, demonstrando a necessária atualização da descrição do curso em relação à atualização do curso e da atuação real, e não projetada, destes engenheiros.
O sombreamento, ou citação constante de termos relacionados à Engenharia Agrícola encontra respaldo na origem do curso e formação do mesmo em outros países.

Ainda se destaca também a necessidade de maior interação entre as diferentes instituições que oferecem o curso, e de seus formandos, para criação de uma definição mais similar e que ajude a clarear do que se trata a profissão e gere maior interesse pelo curso nos vestibulares. 


\section{REFERÊNCIAS BIBLIOGRÁFICA}

\begin{abstract}
AMERICAN SOCIETY OF AGRICULTURAL ENGINEERING. About the Profession. Disponivel em: https://www.asabe.org/About-Us/About-theProfession. Acesso em: 27 ago. 2020.

CUELLO, J. L.; Faces of change, Part 2. Resource: Engineering \& Technology for a Sustainable World 2(2): 10-12, 1995.
\end{abstract}

DUVAL, F. V.; SILVA, F. A. B.; O uso do Twitter como minerador de eventos adversos de medicamentos de combate à malária: o caso da doxiciclina. Cadernos de Saúde Pública, v. 35(5), 2019.

FERGUSON, R.; LOVELL, S. Permaculture for agroecology: design, movement, practice, and worldview. A review. Agronomy for Sustainable Development, v. 34, n. 2, p. 251-274, 2014.

HAN, X.; TIAN, L.; YOON, M.; LEE, M.; A Big Data Model supporting Information Recommendation in Social Network. In: International Conference on Cloud and Green Computing, IEEE computer society, p. 810-813, 2012.

HE, Y.; FANG, H.; FENG, L.; ZHAO, W.; YING, Y.; CUELLO, J.L.; Designing the first Biosystems Engineering program in China. Applied Engineering in Agriculture, Vol. 23(6): 849-855, 2007.
LOEWER, O. J.; A History of Biological Engineering From an Agricultural Engineer's Perspective

(http://www.asae.org/resource/ojloewer.html). Resource 4, 1997.

NUNES, R.; SILVA, C. E. M. V. Biosystems Engineering: unification or redomaining. International Journal of Engineering \& Technology, v. 14, p. 97-101, 2014.

PRODUÇÃO; In: DICIO, Dicionário Online de Português. Porto: 7Graus, 2020. Disponível em: https://www.dicio.com.br/trabalho/. Acesso em: 28/08/2020.

SILVA, C.E.M.V.; NUNES, R.; Redes de Colaboração em Engenharia de Biossistemas no Brasil. Revista Brasileira de Engenharia de Biossistemas, v. 10(2), 181-190, 2016.

TAO, B. Y.; ALLEN, D. K.; OKOS, M. R.; The Evolution of Biological Engineering. International Journal of Engineering Education, v. 22(1), 45-52, 2006.

VISUALIZAÇÕES DA página. , 2020. Disponível em: <http://pageviews.toolforge.org>. Acesso em: 29.08.2020.

YOUNG, R. E.; The bio-type engineering name game. International Journal of Engineering Education, 22(1): 23-27, 2006. 